\title{
Consecuencias de la restricción alimenticia sobre la producción de huevos en hembras tipo Campero INTA*
}

\author{
Pletsch, C.R. ${ }^{1}$; Terraes, J.C. ${ }^{1}$; Revidatti, F.A. ${ }^{1}$; Fernandez, R.J. ; Asiain, M.V. ${ }^{2}$
}

${ }^{1}$ Catedra Producción de Aves, Facultad de Ciencias Veterinarias, Universidad Nacional del Nordeste, Sargento Cabral 2139, Corrientes (3400), Argentina. Tel/fax 03783-425753. E-mail: granja@vet.unne.edu.ar. ${ }^{2}$ Módulo Producción de Aves, Estación Experimental INTA, Las Breñas (Chaco, Argentina).

\begin{abstract}
Resumen
Pletsch, C.R.; Terraes, J.C.; Revidatti, F.A.; Fernandez, R.J.; Asiain, M.V.: Consecuencias de la restricción alimenticia sobre la producción de huevos en hembras tipo Campero INTA. Rev. vet. 20: 2, 86-91, 2009. En el presente trabajo se exponen los resultados obtenidos como respuesta al tratamiento de restricción alimenticia efectuado durante la recría de hembras tipo Campero INTA. Se utilizaron 2.400 aves divididas en cuatro corrales, para un modelo experimental en bloques completamente al azar, en el cual el lote constituyó la unidad experimental y a su vez una repetición simple de cada tratamiento. La cría y recría de ambos sexos se realizó por separado, en compartimientos distintos dentro del mismo galpón semi-abierto. El seguimiento de cada lote tuvo una duración total de 12 meses, correspondiendo cinco meses al período de cría/recría, un mes al de pre-reproducción y seis meses al de postura. El inicio de la postura ( $5 \%$ de producción) se produjo a las 26,5 semanas de vida en aves restringidas, aproximadamente 3 semanas después que las aves controles $(\mathrm{p}<0,05)$. A las 48 semanas de vida, las aves sometidas a restricción produjeron un total acumulado de diez huevos más por ave alojada (99,2 $\pm 9,1$ y $87,7 \pm 10,8$ en grupos restringido y control respectivamente). Las diferencias numéricas existentes para esta variable entre ambos tratamientos fueron manifiestas, pero no alcanzaron significación estadística. Las aves restringidas presentaron una curva típica de producción, constituida por una fase de ascenso rápido, un pico de postura de $81,4 \pm$ $2,8 \%$ (semana 29), una meseta y una caída de puesta progresiva hasta el final del período. El pico de postura alcanzado por el grupo no restringido $(66,7 \pm 4,1 \%)$, presentó un aplanamiento de la curva de producción, con valores inferiores respecto al de las aves restringidas.
\end{abstract}

Palabras clave: gallinas, restricción alimenticia, producción de huevos.

\begin{abstract}
Pletsch, C.R.; Terraes, J.C.; Revidatti, F.A.; Fernandez, R.J.; Asiain, M.V.: Consequences of the nutritional restriction on the production of eggs in Campero INTA hens. Rev. vet. 20: 2, 86-91, 2009. Results obtained in response to food restriction during rearing of CamperoINTA hens, are presented. A total of 2,400 birds were used in four experimental pens, using a generalized randomized block design model in which the flock was the experimental unit and with a simple repetition of each treatment. Rearing and growing of both sexes was conducted separately in different compartments within the same semi-open shed. The monitoring of each lot had a total duration of 12 months, corresponding 5 months to the period of rearing / growing, 1 month to pre-breeding, and 6 months to the reproduction stage. When comparing the onset of laying on each of the regimens tested, there was a delay of approximately 3 weeks in the birds that were subjected to food restriction, reaching $5 \%$ of production at 26.5 weeks $(\mathrm{p}<0.05)$. At 48 weeks, birds subjected to restriction produced a cumulative total of 10 eggs more per housed hen $(99.2 \pm 9.1$ and $87.7 \pm 10.8$ in restricted and control groups, respectively). However, these differences were not significant, although the evident numerical difference between both treatments. Food restricted birds outlined a typical curve path comprising a fast rising phase, an egg production peak of $81.4 \pm 2.8 \%$ (week 29), a plateau and a gradual decrease until the end of treatment. The production peak reached by the unrestricted group $(66.7 \pm 4.1 \%)$, showed a flattening of the production curve with lower values when compared to other treatments.
\end{abstract}

Key words: hens, nutritional restriction, production of eggs.

\footnotetext{
* Fragmento de la tesis del primer autor (Maestría en Producción Animal Subtropical, FCV-UNNE).

Recibido: 17 setiembre 2009 / Aceptado: 21 octubre 2009
} 


\section{INTRODUCCIÓN}

En nuestro país, el Instituto Nacional de Tecnología Agropecuaria (INTA) cuenta con una estructura de producción avícola de tipo piramidal, cuyo esquema se inicia en un núcleo genético que funciona en la localidad de Pergamino (Provincia de Buenos Aires), en el cual se llevan a cabo cruzamientos entre diferentes razas. Desde este lugar, los reproductores padres son enviados a granjas de multiplicación ubicadas en distintos puntos del país donde se llevan a cabo los cruzamientos para la producción de carne aviar (pollo "Campero-INTA"). Debido a su mayor rusticidad, estas aves se adaptan con mayor eficiencia que los híbridos comerciales a las condiciones locales y presentan mejores índices de producción que las aves criollas no seleccionadas, por lo cual constituyen una alternativa económica importante (consumo propio y/o comercialización de excedentes) ${ }^{3}$.

La selección genética para producción de carne dio como resultado un incremento en el peso corporal en varios puntos de la curva de crecimiento de los reproductores; sin embargo también afectó el patrón de crecimiento de distintos tejidos corporales, entre ellos el del aparato reproductor, modificando su funcionamiento (madurez sexual y eficiencia reproductiva) ${ }^{1,13}$. Aunque el peso corporal de la polla al inicio de la postura y su uniformidad son considerados importantes indicadores por parte de los productores, estas variables deben ser consideradas junto a la composición corporal, el patrón de crecimiento previo, la edad cronológica de las aves y su desarrollo genital ${ }^{6,13}$.

Se han hallado correlaciones negativas entre la edad a la madurez sexual con el peso corporal, rendimiento de carne de pechuga y porcentaje de grasa acumulada, aunque se considera necesaria una cantidad mínima de grasa corporal a fin de garantizar un adecuado ciclo ovulatorio. También se han comprobado correlaciones negativas entre peso corporal y rendimiento de carne de pechuga con respecto a la fertilidad ${ }^{13}$.

La gallina reproductora pesada alimentada ad libitum desarrolla un sobrepeso que lleva a disfunciones en la ovulación y oviposición, fenómeno conocido como "síndrome de oviposición errática y huevos defectuosos", en el cual se presentan varias anomalías de carácter reproductivo como atresia folicular, ovulación abdominal, ovulaciones múltiples, huevos de mala calidad (dobles yemas, problemas de cáscara), oviposición en horarios anormales (oviposición errática), asociados con prolapsos de oviducto. Todos estos problemas se traducen en una disminución en la producción de huevos aptos para la incubación, sumado a un aumento de la incidencia de la mortalidad embrionaria y materna, con reducida fertilidad ${ }^{16}$.

Actualmente en las líneas de aves de rápido crecimiento (productoras de carne), se exige que la reproductora pese a las 21 semanas lo que su descendencia pesará a las 6 semanas ${ }^{11}$. Si a estas aves se les permite comer sin límites, alcanzarán un peso incompatible con la producción óptima de semen y de huevos para incu$\operatorname{bar}^{7}$. Dado que dicho problema de correlación negativa entre los rasgos reproductivos y el peso corporal nunca permitieron una solución genética eficaz, por ello es que se plantean soluciones de tipo medioambientales ${ }^{7}$ . El manejo de la alimentación y con ella la asignación de nutrientes, supone considerar múltiples factores y componentes de variada naturaleza, como cantidad y calidad del alimento suministrado, presentación física (harina, granulado, pellets), forma de suministro ( $a d-$ libitum o restricción), tipo y manejo de los comederos, entre otros componentes que hacen al rubro de la nutrición ${ }^{4}$.

En la actualidad se considera que los programas de restricción alimenticia deben garantizar además de un peso corporal determinado, un consumo mínimo de energía y proteína durante la fase de recría si se pretende lograr buenos resultados reproductivos. Se estima que es necesario un consumo mínimo de $22.000 \mathrm{kcal}$ de energía metabolizable y $1.200 \mathrm{~g}$ de proteína bruta por gallina para lograr una buena productividad ${ }^{4}$. Esta es la razón por la cual debe tenerse extremo cuidado al reducir la cantidad de alimento.

El objetivo del presente trabajo fue evaluar los efectos que produce la restricción alimenticia durante la fase de recría, sobre la madurez sexual y producción de huevos en hembras reproductoras tipo CamperoINTA.

\section{MATERIAL Y MÉTODOS}

Los ensayos se llevaron a cabo en el Módulo de Reproducción de Aves de la Estación Experimental del INTA Las Breñas (Chaco, Argentina). Se utilizaron reproductores padres del pollo Campero INTA, ave productora de carne que se caracteriza por su rusticidad y crecimiento más lento que las estirpes híbridas de pollos parrilleros. En el esquema de producción del Campero INTA, el padre es un híbrido simple proveniente del cruzamiento de las razas Cornish colorada y Plymouth Rock blanca y la madre es una cruza de las razas Rhode Island colorada y Anac.

La cría (0 a 5 semanas) se realizó en un galpón cerrado con ventanas y piso de cemento alisado, en el cual se hicieron separaciones para alojar los grupos experimentales, a una densidad de 12 aves por $\mathrm{m}^{2}$. Para la provisión de calor se empleó una campana a gas de $120 \mathrm{~cm}$ de diámetro. Se utilizaron bebederos de plato con recipiente invertido y comederos tolva de $6 \mathrm{~kg}$ de capacidad.

A las 5 semanas de vida (final de la etapa de cría) las hembras fueron trasladadas a un galpón de recría semi-abierto asignando una densidad de 6 aves por $\mathrm{m}^{2}$. Los machos fueron alojados en galpones similares pero a una densidad de 5 aves por $\mathrm{m}^{2}$. En ambos sexos, se utilizaron comederos tolva de $15 \mathrm{~kg}$ y bebederos campana durante la recría (6 a 20 semanas del ciclo).

En ambos tratamientos, el consumo registrado durante la etapa de la cría fue de $1.150 \mathrm{~g}$ de ración, lo cual 
Tabla 1. Composición de la dieta correspondiente tanto al tratamiento 1 como al 2, para las distintas etapas del ciclo.

\begin{tabular}{lcccc}
\hline \multirow{2}{*}{ composición } & \multicolumn{4}{c}{ tipo de alimento } \\
\cline { 2 - 5 } & iniciador & recría & pre-reprod. & reprod. \\
\hline energ.met. kcal/kg & 2900 & 2900 & 2800 & 2850 \\
$\mathrm{Ca}(\%)$ & 0.95 & 0.9 & 2 & 3.5 \\
$\mathrm{P}(\%)$ & 0.45 & 0.4 & 0.5 & 0.5 \\
proteína (\%) & 21 & 19 & 18 & 17 \\
grasa (\%) & 3.2 & 3 & 3 & 3.5 \\
fibra (\%) & 3.5 & 3.3 & 3.3 & 3 \\
ac. linoleico (\%) & 1.3 & 1.3 & 1.7 & 1.7 \\
lisina (\%) & 1 & 0.85 & 0.9 & 0.8 \\
metionina (\%) & 0.5 & 0.45 & 0.4 & 0.4 \\
met.+ cistina (\%) & 0.8 & 0.75 & 0.7 & 0.7 \\
treonina (\%) & 0.8 & 0.7 & 0.7 & 0.65 \\
\hline
\end{tabular}

aportó $241,5 \mathrm{~g}$ de proteína bruta y $3.335 \mathrm{kcal}$ de EM/ $\mathrm{kg}$ en función de la composición química proximal de la ración (Tabla 1).

En la Tabla 1 se observa la composición química de todas las raciones que se suministraron a los reproductores Campero INTA durante el ciclo completo, la cual y como corresponde a la naturaleza del presente ensayo -en el cual la variable independiente se halla asociada a una restricción de tipo cuantitativa- presenta una única composición química.

De igual manera y siempre con los datos proporcionados por la cabaña de referencia (en cuanto al consumo y la calidad de la dieta), se calculó la cantidad de energía y proteína consumidas por las reproductoras pertenecientes al tratamiento 2 (control) durante la recría en $23.913 \mathrm{kcal} \mathrm{EM} / \mathrm{kg}$ y $1.567 \mathrm{~g}$ de proteína bruta para un consumo acumulado de $8.246 \mathrm{~g}$ para esta fase. De esta manera el grupo control consumió $9.396 \mathrm{~g}$ de alimento durante la etapa de cría/recría, que aportaron $27.248 \mathrm{kcal} \mathrm{EM} / \mathrm{kg}$ y $1.809 \mathrm{~g}$ de proteína bruta.

Considerando un consumo acumulado de 8.246 g entre las semanas 5 y 20 , la restricción del $27 \%$ de dicha cantidad equivale al suministro de $6.020 \mathrm{~g}$ de alimento en este período, lo que representa $1.144 \mathrm{~g}$ de proteína bruta y $17.458 \mathrm{kcal} \mathrm{EM} / \mathrm{kg}$ para el tratamiento 1 , en el cual el consumo para la fase cría/recría alcanzó un total de $7.170 \mathrm{~g}$ de proteína bruta que ofrecieron $20.793 \mathrm{kcal} \mathrm{EM} / \mathrm{kg}$ y $1.385 \mathrm{~g}$ de proteína. Esta oferta de nutrientes acumulados mínimos al final de la recría, corresponde a lo propuesto por otros autores que han trabajado con líneas paternas de reproductores pesados de alto rendimiento para la producción comercial de pollos barrilleros ${ }^{4}$.

Tratamientos. El tratamiento "1" consistió en suministrar, a partir del inicio de la recría hasta su final (inicio del fotoestímulo), un 27\% menos de la dieta de recría utilizada habitualmente en el establecimiento (Tabla 2). El tratamiento "2" (estándar o control) consistió en el programa de alimentación basado en las cantidades de alimento sugeridas por la cabaña de origen, ajustadas semanalmente.
Tabla 2. Consumo de alimento en gramos por ave por semana durante la fase de recría, según tratamientos.

\begin{tabular}{ccc}
\hline semana & tratamiento 1 & tratamiento 2 \\
\hline 5 & 256 & 350 \\
6 & 281 & 385 \\
7 & 296 & 406 \\
8 & 307 & 420 \\
9 & 317 & 434 \\
10 & 327 & 448 \\
11 & 337 & 462 \\
12 & 347 & 476 \\
13 & 358 & 490 \\
14 & 383 & 525 \\
15 & 399 & 546 \\
16 & 419 & 574 \\
17 & 450 & 616 \\
18 & 480 & 658 \\
19 & 511 & 700 \\
20 & 552 & 756 \\
\hline total & 6.020 & 8.246 \\
\hline
\end{tabular}

Tabla 3. Lapso transcurrido entre inicio y pico de producción en aves restringidas y controles.

\begin{tabular}{lcl}
\hline tratamiento & edad (semanas) & DE \\
\hline control & 6,7 & 0,5 \\
restringidas & 2,5 & 1,7 \\
\hline
\end{tabular}

Diseño experimental. Se aplicó un modelo en bloque completamente al azar, en el cual el lote constituyó la unidad experimental y a su vez una repetición simple de cada tratamiento. Se utilizaron para este ensayo cuatro lotes de reproductoras de 600 hembras Campero INTA divididas al azar al final de la cría, en dos grupos de 300 aves cada uno, en las cuales se aplicaron los tratamientos. El seguimiento de cada lote tuvo una duración total de 12 meses, correspondiendo 5 al período de cría/recría, 1 al de pre-reproducción y 6 al de postura.

Las "variables respuesta". Al final de la etapa de fotosensibilización fueron: inicio de postura del lote, en este caso, y por cada grupo, se estableció el tiempo necesario para iniciar la fase de postura $(5 \%$ de producción de huevos). En fase de reproducción (entre 20-48 semanas de vida): en el presente trabajo, el análisis de la fase de postura abarca los primeros 6 meses de producción. Durante esta etapa fueron registradas las siguientes variables: edad para alcanzar el pico de postura: definido como aquél en el que se logra el máximo porcentaje de postura del lote; lapso de tiempo transcurrido entre inicio y pico de producción (en semanas); porcentaje de postura máximo alcanzado (pico de postura): se obtuvo dividiendo el promedio de producción de huevos /día puestos en cada semana, por el número de aves alojadas durante el mismo período; número de huevos por gallina durante el período de puesta analizado (hasta las 48 semanas de vida): esta variable fue obtenida mediante el cociente entre el promedio acumulado de huevos y el promedio de aves en producción 
de cada grupo experimental. También fue analizada la curva de postura de cada grupo experimental.

\section{RESULTADOS}

Inicio de postura. $\mathrm{Al}$ comparar el inicio de la postura en las pollas de cada uno de los tratamientos ensayados, se observó una demora de aproximadamente 3 semanas en las aves que fueron sometidas a la restricción alimenticia del $27 \%$; en efecto, en estos lotes se alcanzó el $5 \%$ de postura a las $26,5 \pm 0,5$ semanas de vida, mientras que las aves control lo hicieron a las $23,8 \pm 0,5$ semanas. Estas diferencias fueron estadísticamente significativas $(\mathrm{p}<0,05)$.

Edad para alcanzar el pico de postura. En esta variable se obtuvieron medias aritméticas y desvíos estándares muy similares para ambos tratamientos: semana $30 \pm 0,8$ y semana $29 \pm 1,2$ para tratamientos control y restringido, respectivamente. Dichas diferencias no fueron estadísticamente significativas $(p>0,05)$.

Lapso transcurrido entre inicio y pico de producción. La dinámica del proceso transcurrido en la fase del inicio del fotoestímulo hasta llegar al momento de registrarse el pico de producción, acusó diferencias estadísticas significativas $(\mathrm{p}=0,03)$, como indica la Tabla 3).

Porcentaje de postura máxima alcanzada (pico de postura). Para esta variable de producción se obtuvieron $66,75 \pm 4,1$ y $81,45 \pm 2,8 \%$ para los tratamientos control y restringido respectivamente. Las diferencias constatadas entre tratamientos fueron significativas $(\mathrm{p}<0,05)$.

Número de huevos por gallina durante el período de puesta analizado (hasta las 48 semanas de vida). Los lotes de aves sometidas a restricción alimenticia durante la recría produjeron, en promedio, un total acumulado de 10 huevos más por ave alojada durante el período de postura analizado (inicio de producción a 48 semanas de vida), registrando un total de 99,2 \pm 9,1 y $87,7 \pm 10,8$ huevos/ave para el grupo restringido y control respectivamente. Si bien estas medias no alcanzaron a ser significativas, es dable destacar la diferencia en cantidad de huevos existente entre ambos tratamientos.

Evolución de la curva de postura de cada grupo experimental. En la Figura 1 es posible observar el trazado de las curvas de postura para los distintos tratamientos. Se advierte que las aves sometidas a restricción alimenticia durante la fase de recría, delinearon un trazado de curva típico, constituido por una fase de ascenso rápido, una meseta y un descenso progresivo hasta el final del momento de estudio, en el cual dicho lote acusó un $60 \%$ de puesta. Los lotes no restringidos, presentaron un aplanamiento de la curva de producción con valores inferiores respecto al otro tratamiento.

\section{DISCUSIÓN}

En general, se acepta que la curva de pesos provista por las líneas de origen genético constituye una guía idónea para lograr un adecuado desarrollo físico y genital de un lote al final de la recría. Esto se base en la comprobada correlación existente entre dichas variables con altos rendimientos reproductivos. Sin embargo, en la actualidad, y debido a las diferencias genéticas entre líneas y a los continuos cambios en los rendimientos obtenidos en las aves, se asume que tales curvas han ido perdiendo vigencia, siendo reemplazadas progresivamente por el criterio de la alimentación basada en fijar las cantidades acumuladas mínimas de energía y proteína que deben consumir los reproduc-

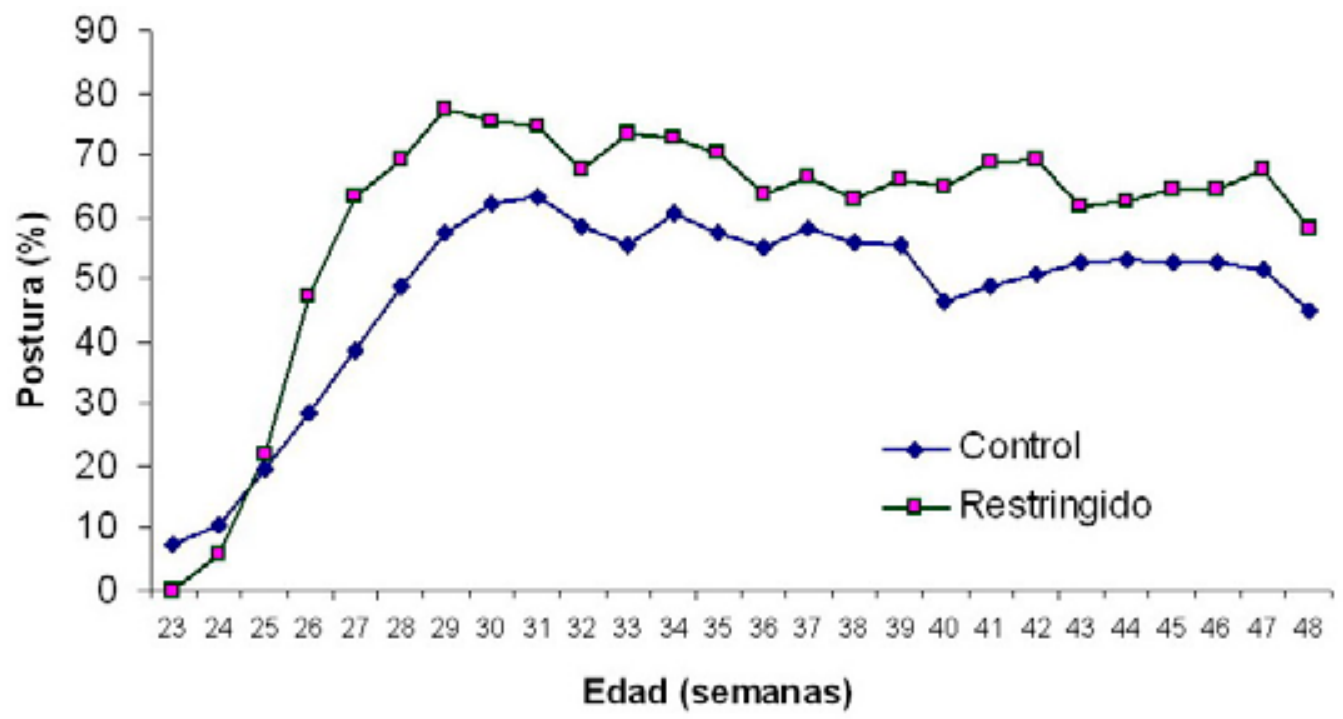

Figura 1. Porcentaje de postura a lo largo del período de estudio según tratamientos. 
tores (machos y hembras) al final del período de recría (o de fotosensibilización) garantizando una optima producción futura ${ }^{4}$.

Teniendo en cuenta la variabilidad que ofrecen las características genéticas de la línea con la que se trabajó aquí (reproductores Campero-INTA) y que la curva de consumo sugerida por la cabaña y la calidad del alimento suministrado ofrecen valores altamente superiores a los recomendados para otras líneas pesadas, es que se planteó en el presente ensayo fijar como variable independiente el ajuste de la cantidad de ración a suministrar para alcanzar así los nutrientes acumulados mínimos con los que trabaja la industria avícola en la actualidad. La lógica indicaría que si aves reproductoras tales como Campero-INTA, relativamente poco seleccionadas por tamaño y velocidad de crecimiento respecto a las híbridas comerciales, consumen un total de nutrientes superiores a éstas (que presentan una mayor masa corporal y tasa de crecimiento), llegarán indefectiblemente con una sobreoferta de nutrientes (tal como lo sugiere el marco teórico existente), conspirando contra su rendimiento reproductivo futuro.

En tal sentido, y a la luz de los resultados hallados aquí en materia de peso corporal, es posible ratificar que al reducir un $27 \%$ las cantidades de alimento suministrado a las pollas en recría, se alcanzan los porcentajes acumulados mínimos tanto de energía como de proteína bruta considerados como óptimos para el rendimiento futuro del lote ${ }^{4,9}$. Se estima necesario un consumo mínimo de $22.000 \mathrm{kcal}$ de energía metabolizable y $1.200 \mathrm{~g}$ de proteína bruta por polla para lograr una buena productividad ${ }^{4,10}$. Estos valores coinciden con lo establecido en el presente ensayo en el tratamiento 1 (restringido), aunque ello haya provocado una reducción relativa en el peso corporal de las aves no restringidas.

Varios ensayos realizados en reproductores pesados sometidos a programas de restricción concluyen que bajo ciertas circunstancias de manejo, es necesario que transcurran varias semanas para apreciar la respuesta a una restricción determinada. En algunos casos se citan períodos de hasta 3 o 4 semanas hasta detectar, mediante la curva de pesos, la respuesta al programa instaurado ${ }^{8}$. En el caso particular del presente trabajo, las diferencias numéricas comenzaron a insinuarse recién después de transcurridas unas 7 semanas de iniciada la restricción (semana 12 del ciclo), para consolidarse finalmente hacia la semana 16. En tal sentido, las aves de más rápido crecimiento (líneas parentales de parrilleros) responden más intensamente al grado de restricción que las líneas poco seleccionadas para ese parámetro ${ }^{11}$, tal como las aquí utilizadas.

A diferencia de lo registrado en el presente trabajo, en el cual el grado de restricción cuantitativa durante la recría se tradujo en una merma manifiesta del peso promedio de las pollas, algunos autores no obtuvieron respuestas a la restricción en términos de descenso del peso corporal y atribuyen este hecho a que en algunos casos las aves sometidas a restricción, compensan la disminución de la cantidad de alimento que se les suministra incrementando la eficiencia de conversión a través de un mejora en la digestión de la materia seca ${ }^{2}$. Sin embargo, es más probable que el mantenimiento del peso promedio de la población ocurra a expensas de un deterioro de los porcentajes de uniformidad ${ }^{5}$, tal como se ha constatado en este ensayo.

Adicionalmente, al estudiar la curva de crecimiento obtenida con el programa de restricción implementado se puede constatar que la misma (en particular a partir de las 16 semanas del ciclo), adopta una fase de ascenso final, lo cual es considerado por la mayoría de los autores como un objetivo esencial a lograr, en particular cuando se lleva a cabo un programa de restricción ${ }^{12}$, ${ }^{13}$. Esto último se debe al hecho de que en realidad es necesario llegar al final de la recría no solo con los nutrientes acumulados mínimos sino con un peso corporal adecuado (tal como se ha conseguido en el presente trabajo), dando hacia el final de la recría una curva en ascenso.

El éxito en la producción futura de estas aves depende del grado de reservas nutricionales que las mismas dispongan al final del período de fotosensibilización ${ }^{14}$. Por ende no es conveniente fotoestimular un lote a edad temprana (antes de las 20 semanas) o que no haya alcanzado el peso corporal recomendado por la línea de origen para dicha edad, o -más actualmentela cantidad de nutrientes acumulados mínimos necesarios, caso contrario el inicio de la actividad reproductiva no ocurrirá en forma apropiada y comprometerá el rendimiento futuro del lote ${ }^{15,16}$. Hemos constatado aquí diferentes pesos corporales a las 20 semanas según tratamientos, pero con trazados de curvas de crecimiento similares y esto (sumado al ajuste de la cantidad de nutrientes ofrecidos durante la recría) permitió proyectar diferencias en el comportamiento productivo entre los tratamientos, hecho que ha sido constatado mediante los resultados obtenidos en la mayor parte de las variables reproductivas analizadas.

A la luz de los resultados productivos logrados en este trabajo, resulta evidente que el nivel de restricción aplicado puede considerarse adecuado, en coincidencia con lo que sostienen algunos autores en el sentido que el grado o intensidad de restricción puede lograr los pesos deseados pero no obstante derivar en estrés, que tendrá derivaciones negativas tanto en el desarrollo corporal como genital del ave en el inicio de la etapa de postura, con las consecuencias correspondientes en el desempeño reproductivo ${ }^{21}$. Tales consecuencias no fueron constatadas en el presente ensayo.

Existe abundante marco teórico que da soporte a los mejores resultados obtenidos en relación a los distintos índices de producción de huevos que se lograron en las aves restringidas respecto a las no restringidas. En principio, se ha corroborado la correlación negativa existente entre la edad a la madurez sexual e inicio de producción con respecto al peso corporal ${ }^{18}$. En tal sentido nuestros resultados coinciden con lo hallado por autores que no solo observaron mejores rendimien- 
tos reproductivos en aves de tipo pesado cuando ellas eran restringidas, sino también un menor peso corporal, rendimiento de carne de pechuga y porcentaje de grasa acumulada ${ }^{13}$. En este reporte se considera necesaria una cantidad mínima de grasa a fin de garantizar un adecuado ciclo ovulatorio.

En el presente trabajo se ha corroborado que, tal como se sostiene en el marco teórico, aunque la restricción alimenticia provoque una demora en el inicio de la madurez sexual y concomitantemente en el inicio de la postura, esto se compensa con la mejora en la tasa de postura ${ }^{14}$, en una disminución de la producción de "huevos de polla" (que no son viables para la incubación) y en un incremento en la persistencia de la postura ${ }^{15,19-21}$. Por ello, el grado de restricción es fundamental ya que si es excesivo puede derivar en estrés, acarreando consecuencias negativas tanto en el desarrollo corporal y genital del ave como en el desempeño reproductivo ${ }^{16,21}$.

El conclusión, los resultados obtenidos en el presente trabajo indican que los reproductores CamperoINTA respondieron de manera manifiesta a la restricción alimenticia cuantitativa, hecho puesto en evidencia mediante la merma en los promedios de peso corporal y mejores índices productivos en las aves tratadas. De hecho, ello constituye un hallazgo a destacar, ya que independientemente de los resultados zootécnicos a los que se pueden arribar a posteriori (y que constituyen precisamente el sentido de la restricción alimenticia en cantidad), no siempre las aves restringidas manifiestan un descenso concomitante en los promedios de peso, ya que en algunos casos aumenta la eficiencia en la asimilación de nutrientes. A partir de los resultados productivos hallados aquí, es posible concluir que los reproductores Campero-INTA respondieron a los niveles de nutrientes acumulados mínimos de manera semejante a las líneas parentales productoras del pollo parrillero.

\section{REFERENCIAS}

1. Barbato GF. 1999. Genetic relationships between selection for growth and reproductive effectiveness. Poultry Sci 78: 444-452.

2. Bennett CD, Leeson S. 1989. Growth of broiler breeder pullets: skip-a-day versus daily feeding. Poultry Sci 68: 836-838.

3. Bonino MF, Canet ZE. 1999. Producción de pollos y huevos camperos, Boletín Técnico editado por la Dirección de Comunicaciones INTA, Buenos Aires, 39 p.

4. Brake JT. 2000. Ciencia y arte del manejo de reproductoras pesadas. Anales V Seminario de Actualización Avícola (AMEVEA), Colón, Entre Ríos (Argentina), p. 183-220.

5. Bramwell K. 2003. Breeder flock uniformity can make a difference. Disponible en: http://poultryandeggnews.com/ poultrytimes/focus/july2003/608568.html.

6. Joseph NS, Robinson FE, Renema RA, Thorsteinson KA. 2003. Comb growth during sexual maturation in female broiler breeders. J Appl Poult Res 12: 7-13.
7. Kerr CL, Hammerstedt RH, Barbato GF. 2001. Effects of selection for exponential growth rate at different ages on reproduction in chickens. Avian \& Poultry Biol Rev 12: 127-136.

8. Kirby JD, Froman DP. 2000. Reproduction in the male birds. In: Sturkie's Avian Physiology (Causey G, Hittow W. Ed.), 5th ed., Academics Press, San Diego (USA), 597 p.

9. Moye, J. 1993. Fine-tuning. The Breeder Male. CobbVantress Tech News 1: 2-4.

10. Peak SD, Brake J. 1994. A comparison of pullets body weight and nutrients consumption patterns as indicators of potential reproductive performance of broiler breeders. Poultry Sci 73: 1-3.

11. Piquer J. 1999. Interaccion nutrición-reproducción en aves. Public. FEDNA (españa), on line: www.etsia.upm.es/ fedna/capitulos/2001CAPIV.

12. Poole D. 2003. Una visión práctica de la nutrición de reproductoras pesadas adultas y manejo de los alimentos en EE.UU. Rev. Selec Avic 45: 817-822.

13. Reddish JM. 2004. Evaluation of the effects of selection for increased body weight and increased yield on growth and development of Poultry. Tesis PhD, Ohio State University (USA), $111 \mathrm{p}$.

14. Renema RA, Robinson FE, Proudman JA, Newcombe M, McKay RI. 1999. Effects of body weight and feed allocation during sexual maturation in broiler breeder hens. 2 . Ovarian morphology and plasma hormone profiles. Poultry Sci 78: 629-639.

15. Robinson FE, Wilson JL, Yu MW, Fasenko GM, Hardin RT. 1993. Effects of feed allocation on growth and carcass traits in three strains of broiler breeder females. Can J Anim Sci 71: 912-922.

16. Robinson FE, Renema RA. 2003. Managing what you can't see: the role of feed in breeder ovary management. Conference, University of Alberta (Canada). On line: www.poultryindustrycouncil.ca/bbreedersession.pdf.

17. Robinson FE, Renema RA. 2003. Variation between strains of breeders in managing sexual maduration. Conference, University of Alberta (Canada). On line: www. poultryindustrycouncil.ca/bbreedersession.pdf.

18. Souza PA, Souza HB, Brognoni E, Rocha OE. 1994. Influência da idade da ave sobre a qualidade do ovo. Científica (São Paulo, Brasil) 22: 217-226.

19. Walsh TJ. 1996. The effects of nutrition and feed programmes on reproductive performance and fertility of broiler breeders. Tesis PhD, North Carolina State University, Raleigh (USA), $154 \mathrm{p}$.

20. Widowski TM, Wong LF, Duncan IJ. 1998. Rearing with males accelerates onset of sexual maturity in female domestic fowl. Poultry Sci 77: 150-155.

21. Yu M, Robinson W, Charles FE, Weingardt R. 1992. Effect of feed allowance during rearing and breeding on female broiler breeders. 2. Ovarian morphology and production. Poultry Sci 71: 1750-1761. 